\title{
Computation of Reliability and Bayesian Analysis of System Reliability for Mukherjee Islam Failure Model
}

\author{
Shas hi Saxe na", Shazia Zarrin, Mus tafa Kamal, Arif-Ul-Is lam
}

Department of Statistics \& Operation Research Aligarh Muslim University, Aligarh, India

\begin{abstract}
The Mukherjee Islam Failure Model is considered as a simple model to assess component reliability and may exhibit a better fit for failure data and also provide more appropriate information about hazard rate. This paper presents the reliability computation and Bayesian estimation of system reliability when the applied stress and strength follows the Mukherjee Islam Failure Model. The results obtained in this paper may be applied to semiconductor devices. The main objective of this paper is to compute reliability and Bayesian analysis of system reliability. Maximum likelihood estimator and Bayesian methods are used inside the paper.
\end{abstract}

Keywords Stress-Strength Model, Bayes Estimation, Mukherjee Islam Failure Model, Maximum likelihood Estimator, Reliability Computation

\section{Introduction}

Reliability is a broad term that focuses on the ability of a product to perform its intended function. The word "reliability" refers to the ability of a system to perform its stated purpose adequately for a specified period of time under the operational conditions encountered. A system is said to be absolutely reliable if some undesirable events, called failures, do not occur in the system's operation. Every system has its own set of such undesirable events. The system defined here could be an electronic or mechanical hardware product, a software product, a manufacturing process or even a service. For example, for a mechanical system, a failure is a breakdown of some of its parts or an increase in vibration above the permitted level. One of the most dangerous failures of a nuclear reactor is a leak of radioactive material. The reliability characteristics are usually expressed in terms of the lifetime. The lifetime is a random time from the beginning of the operation until the appearance of a failure, after which further operation is impossible.

The concept of reliability is used in a variety of business and industrial settings. In general, the concept of re liability is applied where it is important to achieve the same results over and over again. A manufacturing process is said to be reliable when it achieves the same result, with in definite limits, each time it occurs. An automobile, or other type of product, is reliable if it performs consistently and up to

* Corresponding author:

sshashisaxena@gmail.com (Shashi Saxena)

Published online at http://journal.sapub.org/ajms

Copyright (C) 2012 Scientific \& Academic Publishing. All Rights Reserved expectations.

Statistical distributions have long been employed in the assessment of semiconductor device and product reliability. The use of the exponential distribution which is frequently preferred over mathematically more complex distribution, such as the Weibull and the lognormal a mong others, suggest that most engineers favor the application of simpler model to obtain failure rates and reliability figures quickly. It is therefore proposed that the Mukherjee Islam Failu re Model be considered as a simpler alternative which, in some circumstances, may exhibit a better fit for failure data and provide more appropriate information about reliability and hazard rates. The Mukherjee Islam Failu re model is also used for appropriate representation of the lower tail of the distribution of random variable having fixed lower bound.

In the context of 'strength-reliability', the stress-strength model describes the life of a component, which has a random strength $Y$ and is subjected to a random stress $X$. The component fails at the instant that the stress applied to it exceeds the strength, and the component will function satisfactorily whenever $Y>X$. Thus, $(Y>X)$ is a measure of component reliability. It has many applications in engineering concepts, deterioration of rocket motors, static fatigue of ceramic components, fatigue failure of aircraft structure and the aging of concrete pressure vessels. Maroof and Islam ${ }^{1}$ consider the problem of Bayesian estimation of system reliability using the Lo max model. Arulmo zhi $i^{2}$ gives a simp le and efficient computational method for determining the system reliability of k-out-of-n systems having unequal and equal reliabilities for components. Kucheryavyi and Mil'kov ${ }^{6}$ consider the problem of strength reliability computation for a beam section of a main gas pipeline.

In this paper we compute the reliability and also obtain the Bayesian estimation of a system's reliability when the 
applied stress and strength follow the probability distribution of Mukherjee Islam Failure.

\section{The Model}

Let us consider the Mukherjee Islam Failure model with probability density function (pdf)

$$
f(x)=\frac{p}{\theta^{p}} x^{p-1}, \quad 0<x<\theta, p>0, \theta>0
$$

and the cumulative distribution function (cdf)

$$
F(x)=\left(\frac{x}{\theta}\right)^{p} \quad 0<x<\theta, p>0, \theta>0
$$

where $\theta$ is the scale parameter and $p$ is the shape parameter. The above distribution mainly appears as the inverse distribution of the Pareto distribution.

The theoretical mean $(\mu)$ and variance $\left(\sigma^{2}\right)$ are derived as:

$$
\begin{gathered}
\mu=\frac{p \theta}{p+1} \\
\sigma^{2}=\frac{p \theta^{2}}{(p+2)(p+1)^{2}}
\end{gathered}
$$

The corresponding survival function is

$$
S(x)=1-\left(\frac{x}{\theta}\right)^{p}
$$

The hazard function of $x$, denoted as $h(x)=f(x) / S(x)$ is obtained as

$$
h(x)=\frac{\frac{p x^{p-1}}{\theta^{p}}}{1-\left(\frac{x}{\theta}\right)^{p}}=\frac{p x^{p-1}}{\theta^{p}-x^{p}}
$$

Time to failure is given by

$$
F(t)=1-\exp \left[-\int_{0}^{t} h(x) d x\right]=1-\exp \left[-\int_{0}^{t} \frac{p x^{p-1}}{\theta^{p}-x^{p}} d x\right]
$$

Integrating out by substituting $\theta^{p}-x^{p}=u$, we get

$$
\begin{aligned}
F(t)= & -\exp \left[-\int_{\theta^{p}}^{\theta^{p}-t^{p}} \frac{1}{u} d u\right]=1-\exp [\log u]_{\theta^{p}}^{\theta^{p}-t^{p}} \\
& =1-\exp \left[\log \left(\theta^{p}-t^{p}\right)-\log \theta^{p}\right] \\
& =1-\exp \left[\log \frac{\theta^{p}-t^{p}}{\theta^{p}}\right]=1-\frac{\theta^{p}-t^{p}}{\theta^{p}}
\end{aligned}
$$

\section{Maximum Likelihood Estimator (MLE)}

Let $x_{1}, x_{2}, \ldots x_{n}$ be a random sample of size $n$. We assume that the lifetime of these components follows Mukherjee Islam Failure model, and then the likelihood function is given by

$$
L(p, \theta)=\prod_{i=1}^{n}\left[\frac{p}{\theta^{p}} x_{i}\right]=\frac{p^{n}}{\theta^{n p}} \prod_{i=1}^{n} x_{i}^{p-1}
$$

Then log-likelihood function is

$$
\ln L(p, \theta)=n \ln p-n p \ln \theta+(p-1) \sum_{i=1}^{n} \ln x_{i}
$$

Therefore the MLE's of $\theta$ and $p$ which maximizes eq. (4) must satisfy the normal equations given by

$$
\begin{gathered}
\frac{\partial \ln L(p, \theta)}{\partial p}=\frac{n}{p}-n \ln \theta+\sum_{i=1}^{n} \ln x_{i} \\
\frac{\partial \ln L(p, \theta)}{\partial p}=-\frac{n p}{\theta}=0
\end{gathered}
$$

From eq. (5) we obtain MLE of $p$ as a function of $\theta$ given by

$$
\hat{p}=\frac{n}{n \ln \theta-\sum_{i=1}^{n} \ln x_{i}}
$$

Fro m eq. (6), we estimate MLE of $\theta$ as follows

$$
\begin{aligned}
& -\frac{n p}{\theta}=0 \\
& \Rightarrow \hat{\theta}=\infty
\end{aligned}
$$

Obviously, it is an absurd result.

In this case we locate MLE as follows:

we have to choose $\theta$ so that $L(p, \theta)$ in eq. (3) is maximum. Now $L(p, \theta)$ is maximum if $\theta$ is minimum.

Let $x_{(1)}, x_{(2)}, \ldots, x_{(n)}$ be a random sample of size ' $n$ ' independent observation from the given population so that $0 \leq x_{(1)} \leq x_{(2)} \leq \ldots \leq x_{(n)} \leq \theta$.

$$
\Rightarrow \theta \geq x_{(n)}
$$

Since the minimum value of $\theta$ consistent with the sample is $x_{(n)}$, the largest sample observation, $\hat{\theta}=x_{n}$

$\therefore M L E$ for $\theta=x_{(n)}=$ The largest sample observation.

\section{Reliability Computation}

Let $\mathrm{Y}$ represents the strength of an item with density function

$$
f(y)=\frac{p_{1}}{\theta^{p_{1}}} y^{p_{1}-1}, \quad 0<y<\theta, p_{1}>0, \theta>0
$$

where $\theta$ is the scale parameter and $p_{1}$ is the shape parameter .Similarly,

$$
g(x)=\frac{p_{2}}{\theta^{p_{2}}} x^{p_{2}-1}, \quad 0<x<\theta, \quad p_{2}>0, \theta>0
$$

is the probability density function of stress $X$, that the item may suffer where $p_{2}$ and $\theta$ are the shape and scale parameters respectively.

Beg and Singh $^{3}$ (1979) gave estimation of $\mathrm{P}(\mathrm{X}>\mathrm{Y})$ for Pareto distribution. Nandi and $\mathrm{Aich}^{4}$ (1994) have shown that 
Reliability (R) can be obtained as the Laplace transform of the stress and Kotz et. al. ${ }^{5}$ gave the generlization of stess strength model. Their main results can be summarized as follows:

Let $X$ and $Y$ be two non-negative and continuous random variable having densities $f(x)$ and $g(y)$ respectively.

If $X$ and $Y$ independent then probability that $Y$ exceeds $X$ is given by

$$
R=P[y>x]=\int_{0}^{\infty} x f(x)\left\{\int_{1}^{\infty} g(v x) d v\right\} d x
$$

Using eq.(7), we have

$$
\begin{aligned}
R & =P[y>x]=\int_{0}^{\theta} x \frac{p_{1}}{\theta^{p_{1}}} x^{p_{1}-1}\left\{\int_{1}^{\theta} \frac{p_{2}}{\theta^{p_{2}}}(v x)^{p_{2}-1} d v\right\} d x \\
& =\int_{0}^{\theta} x \frac{p_{1}}{\theta^{p_{1}}} x^{p_{1}-1}\left\{\int_{x}^{\theta x} \frac{p_{2}}{\theta^{p_{2}}} t^{p_{2}-1} \frac{d t}{x}\right\} d x \\
& =\int_{0}^{\theta} x \frac{p_{1}}{\theta^{p_{1}}} x^{p_{1}-1}\left\{\frac{p_{2}}{\theta^{p_{2}}}\left|\frac{t^{p_{2}}}{p_{2}}\right|_{x}^{\theta x}\right\} d x \\
& =\int_{0}^{\theta} x \frac{p_{1}}{\theta^{p_{1}}} x^{p_{1}-1} \frac{1}{\theta^{p_{2}}}\left[(\theta x)^{p_{2}}-x^{p_{2}}\right] d x \\
& =\frac{p_{1}\left(\theta^{p_{2}}-1\right)}{\theta^{p_{1}+p_{2}}} \int_{0}^{\theta} x^{p_{1}+p_{2}-1} d x \\
& =\frac{p_{1}\left(\theta^{p_{2}}-1\right)}{\theta^{p_{1}+p_{2}}}\left|\frac{x^{p_{1}+p_{2}}}{p_{1}+p_{2}}\right|_{0}^{\theta} \\
& =\frac{p_{1}\left(\theta^{p_{2}}-1\right)}{\theta^{p_{1}+p_{2}}} \frac{\theta^{p_{1}+p_{2}}}{p_{1}+p_{2}} \\
& =\frac{p_{1}\left(\theta^{p_{2}}-1\right)}{p_{1}+p_{2}}
\end{aligned}
$$

\section{Remark}

If stress and strength have same scale parameter $\theta$, then it shows that in defining the life of a component initial value of $\theta$ doesn't matter.

\section{Bayesian Analysis of System Reliability}

Life time data are important in re liability analys is classical reliability estimation is based on precise lifetime data. Bayesian method has proved to be very useful when the sample size is small. Hierarchical models are one of the central tools of Bayesian analysis. They after many advantages including the ability to borrow strength to estimate individual parameters and the ability to specify complex models that reflect engineering and physical realities. Broadly, Bayesian models have two parts, first the likelihood function and the other is prior distribution. The likelihood function is constructed from the sampling distribution of the data which describe the probability of observing the data before the experiment is performed. The prior distribution describes the uncertainty about the parameters of the likelihood function. The prior distribution is then updated to the posterior distribution after observing the data.

Bayesian approach helps the reliability engineers and participators to incorporate a wide variety of forms of information in the estimation process and the uncertainties in the parameters due to the lack of knowledge are expressed via probability distribution. This is the major departure from the classical methods, since for classical methods all the parameters are true unknown values. There are uncertain parameters being estimated. In Bayesian Analysis the starting point is the choice of priors. The conjugate and the uniform priors are taken as solution commonly. As a rule, conjugate priors lead to straight forward mathematical calculations and because of this reas on it has been applied for reliability estimation.

Let $x_{1}, x_{2}, \ldots x_{n}$ be a random sample drawn from Muher jee Islam Failure model with parameters $\left(\theta, p_{1}\right)$ then their like lihood function is

$$
L\left(x, \theta, p_{1}\right)=\frac{p_{1}^{n}}{\theta^{n p_{1}}} \prod_{i=1}^{n} x_{i}^{p_{1}-1}, \quad p_{1}>0, \theta>0, x>0
$$

Assume the prior distribution of $\theta$ and $p_{1}$ are respectively

$$
g_{1}(\theta)=\frac{1}{\theta}, \quad \theta>0
$$

and

$$
g_{1}\left(p_{1}\right)=\frac{\beta_{1}^{k_{1}} p_{1}^{k_{1}-1} e^{-\beta_{1} p_{1}}}{\Gamma\left(k_{1}\right)}, \quad p_{1}>0, \beta_{1}, k_{1}>0
$$

Let the parameters $\theta$ and $p_{1}$ are independent, then the joint prior of $\theta$ and $p_{1}$ will be

$$
g_{1}\left(\theta, p_{1}\right) \propto \frac{1}{\theta} p_{1}^{k_{1}-1} e^{-\beta_{1} p_{1}}, \quad \theta>0, p_{1}>0
$$

Let combine the likelihood function eq.(8)and the prior density eq.(9) to obtain the posterior as follows:

$$
\prod\left(\theta, p_{1} \mid x\right) \propto \frac{1}{\theta^{n p_{1}+1}} p_{1}^{\delta_{1}-1} e^{-z(n, x)} e^{-p_{1}\left(\beta_{1}-z(n, x)\right)}
$$

where $\delta_{1}=n+k_{1}, z(n, x)=\sum_{i=1}^{n} \ln \left(x_{i}\right)$

Similarly $y_{1}, y_{2}, \ldots y_{l}$ be a random variable from Mukherjee Islam Failure model with parameters $\left(\theta, p_{2}\right)$ then their likelihood function is

$$
L\left(y, \theta, p_{2}\right)=\frac{p_{2}{ }^{l}}{\theta^{l p_{2}}} \prod_{i^{\prime}=1}^{l} y_{i^{\prime}}{ }^{p_{2}-1}, p_{2}>0, \theta>0, y>0
$$

Now the jo int prior distribution of $\theta$ and $p_{2}$ are given by

$$
g_{2}\left(\theta, p_{2}\right) \propto \frac{1}{\theta} p_{2}^{k_{2}-1} e^{-\beta_{2} p_{2}}, \quad \theta>0, p_{2}>0
$$


Hence the posterior density of $\theta$ and $p_{2}$ will be

$$
\prod\left(\theta, p_{2} \mid y\right) \propto \frac{1}{\theta^{l p_{2}+1}} p_{2}{ }^{\delta_{2}-1} e^{-z(l, y)} e^{-p_{2}\left(\beta_{2}-z(l, y)\right)}
$$

where $\delta_{2}=l+k_{2}, z(l, y)=\sum_{i^{\prime}=1}^{n} \ln \left(y_{i^{\prime}}\right)$

Assuming that $p_{1}$ and $p_{2}$ are independent from eq.(10) and eq.(13), the joint bivariate prior density of $p_{1}, p_{2}$ and $\theta$ will be

$$
\begin{aligned}
\prod\left(p_{1}, p_{2}, \theta / x, y\right) \propto \frac{1}{\theta^{n p_{1}+l p_{2}+2}} p_{1}^{\delta_{1}-1} p_{2}^{\delta_{2}-1} \\
e^{-[Z(n, x)+Z(l, y)]} e^{-p_{1}[S(n)]-p_{2}[S(l)]}
\end{aligned}
$$

where $S(n)=\beta_{1}-z(n, x)$ and $S(l)=\beta_{2}-z(l, y)$

It is not easy to evaluate Bayes estimation of reliability but it can be obtained using Mathcad

\section{Example}

A k-out-of-n-System

This system is operational if and only if at least $\mathrm{k}$ component out of the system's n components are operational. An example might be a radar network having n radar control stations which can cover a certain area under control if and only if at least $\mathrm{k}$ of its stations are operational. The structure function is

$$
\varphi(x)=\left\{\begin{array}{lc}
1 \quad \text { if } \sum_{i=1}^{n} x_{i} \geq k \\
0 \quad \text { otherwise }
\end{array}\right.
$$

A particular case of this system when $k=n$ is called a series system. It is operational if and only if all of its elements are up. One can check easily that the structure function for a series system can be represented as

$$
\varphi(x)=\prod_{i=1}^{n} x_{i}=\min _{1 \leq i \leq n} x_{i}
$$

We can think of a series system as an information transmission system having $n$ "stations" in which the information is transmitted properly only if all the stations are operational. Another extreme case of $k$-out-of- $n$ system is $k$ $=1$. Such a system is called parallel system. We can think of a parallel system as a system with one main operating unit and $(n-1)$ extra units in stand by. The system is down if and only if all $n$ units are not operational. The structural function for a parallel system is

$$
\varphi(x)=1-\prod_{i=1}^{n}\left(1-x_{i}\right)=\max _{1 \leq i \leq n} x_{i}
$$

\section{Conclusions}

The Bayesian procedure is considered for determining the reliability of a system of independent parameters. The procedure uses both test and prior data at the component and system levels. It was proposed that the Mukherjee Islam Failure model may be used as a simple model to assess component reliability. The advantage of this distribution is that the hazard rate, as opposed to the exponential distribution's, is non constant and may therefore be more useful in predicting the actual stages of component's life. It is therefore suggested that this distribution be given consideration when analyzing electronic failure data.

\section{REFERENCES}

[1] A. K. Maroof and H. M. Islam, Reliability Computation and Bay esian Analy sis of System Reliability with Lomax Model, The Journal of The Safety And Reliability Society 29(1) (2009) 5-14.

[2] G. Arulmozhi, Direct Method for Reliability Computation of k-out-of-n: Systems, Applied Mathematics and Computations 143 (2003) 421-429.

[3] M. A. Beg and N. Singh, Estimation of $P(X>Y)$ for the Pareto Distribution. IEEE Trans.Reliab. 28 (1979) 411-414.

[4] S. B. Nandi and A. B. Aich, A Note On Estimation Of $P(X>Y)$ For Some Distributions Useful In Life Testing, IAPQR Trans. 19(1) (1994) 35-44.

[5] S. Kotz, Y. Lumelskii and M. Pensky, The Stress Strength Model and its Generalizations, Theory and Applications, World Scientific, Singapore, (2003)

[6] V. I. Kucheryavyi and S. N. Mil'kov, Strength Reliability Computation for a Beam Section of a Main Gas Pipeline. Journal of Machinery Manufacture and Reliability 37(6) (2008) 618-622. 